\begin{tabular}{|c|l|}
\hline Title & Manipulation of supramolecular 2D assembly of functional dy es toward artificial light-harvesting systems \\
\hline Author(s) & Ishida, Yohei \\
\hline Citation & $\begin{array}{l}\text { Pure and applied chemistry, 87(1), 3-14 } \\
\text { https://doi.org/10.1515/pac-20140906 }\end{array}$ \\
\hline Issue Date & 2015-01 \\
\hline Doc URL & http://hdl.handle.net/2115/60350 \\
\hline Rights & The final publication is available at www.degruyter.com \\
\hline Rights(URL) & http://www.degruyter.com/dg/page/308/copyright-agreement \\
\hline Type & Ishida.pdf \\
\hline
\end{tabular}

Instructions for use 
Invited paper

Yohei Ishida*

\title{
Manipulation of supramolecular 2D assembly of functional dyes toward artificial light- harvesting systems
}

\begin{abstract}
In recent years, excellent research has revealed that light-harvesting systems (LHSs) are composed of beautifully aligned chlorophyll molecules; the regulated alignment of chlorophylls is responsible for the efficient and selective light-harvesting energy transfer processes in purple bacteria. This finding led to the construction of a regularly arranged assembly of functional dyes as a step toward fabricating artificial LHSs. While most approaches toward the construction of dye assemblies have depended on molecular interactions such as covalent, coordination, and hydrogen bonds, my approach involves guest-host interactions using an inorganic nanosheet as the host material. This short review presents the construction of a 2D dye assembly and its effective utilization in artificial light-harvesting applications. Owing to the highly stable and uniform 2D alignment of functional dyes on inorganic nanosheets, nearly $100 \%$ singlet-singlet energy transfer and efficient light-harvesting were achieved. I believe that the results presented herein will contribute to the construction of efficient photochemical reaction systems in supramolecular host-guest assemblies, which may facilitate the realization of artificial photosynthesis.
\end{abstract}

Keywords: artificial photosynthesis; inorganic organic hybrid; IUPAC-SOLVAY International Award for Young Chemists; light-harvesting system.

DOI 10.1515/pac-2014-0906

\section{Introduction}

It is widely recognized that humans will face an energy crisis within the next several decades due to the shortage of fossil fuels. Among the various potential solutions, artificial photosynthesis represents a viable option [1]. Photosynthesis in plants is one of the most important photo-energy conversion reactions on earth. Artificial photosynthesis can be defined as a light-driven system that transports electrons from water molecules to an appropriate electron-accepting system, and consists of a photo-induced substrate conversion reaction system such as $\mathrm{O}_{2}$ evolution from water and a light-harvesting system (LHS) [1]. There is some excellent research regarding artificial photo-induced substrate conversion reaction systems such as $\mathrm{O}_{2}$ evolution via the oxidation of water by ruthenium complexes [2], and $\mathrm{CO}$ evolution via the reduction of $\mathrm{CO}_{2}$ by rhenium complexes [3]. A bottleneck for the realization of artificial photosynthesis based on multielectron conversion reactions (such as $\mathrm{O}_{2}$ evolution from water splitting via 4-electron conversion [4]) is the low photon-flux density of sunlight. Because oxidized or reduced catalyst species have typically small extinction coefficients

Article note: A collection of invited, peer-reviewed articles by the winners of the 2014 IUPAC-SOLVAY International Award for Young Chemists.

*Corresponding author: Yohei Ishida, Division of Material Science and Engineering, Faculty of Engineering, Hokkaido University, Kita 13, Nishi 8, Kita-ku, Sapporo, Hokkaido 060-8628, Japan, e-mail: ishida-yohei@eng.hokudai.ac.jp 
and short lifetimes (of $\mu$ s to ms time scales [5]) and will decompose or deactivate before accepting or donating the next electron, multielectron conversion reactions are typically impossible under normal sunlight without an LHS except for some molecular catalyst with long lifetimes [3]. Therefore, the realization of artificial LHSs is essential to generate artificial photosynthesis based on multielectron conversion reactions, especially for the molecular catalysts with short lifetimes of oxidized or reduced species. It should be noted that most of these catalytic performance have been carried out under strong lab light source and thus should be much inefficient under sunlight.

In recent years, excellent research [6] has revealed that LHSs are composed of beautifully aligned chlorophyll molecules; the regulated alignment of chlorophylls is responsible for the efficient and selective lightharvesting energy transfer processes in purple bacteria. In the energy transfer process, the excited energy is transferred to the reaction center on thepico- or femto-second timescale at almost $100 \%$ of the reaction quantum yield [5]. This finding led to the construction of a regularly arranged assembly of functional dyes in an effort to generate an artificial LHS.

To construct a biomimicking artificial LHS, two parameters should be considered: (i) the selection of appropriate dyes and (ii) the method to construct a regularly arranged dye assembly. In regard to (i), many dyes with large extinction coefficients in the visible light region, including porphyrins, phthalocyanine, metal (Ru, Ir, etc.) complexes, perylenebisimide, naphthalenebisimide, and rhodamine, have been utilized as a constituent of artificial LHSs [7-12]. Since each dye has a specific absorption region and is different from other dyes, appropriate combinations of these dyes and/or with other molecules have been investigated. The method for (ii) involves dye assembly, which can be done by (a) covalent bonds [13-15], (b) supramolecular interaction between dyes [16-19], or (c) supramolecular interaction between dyes and inorganic materials [20-22].

Förster proposed the theory of Förster resonance energy transfer in the 1940s [23]; three decades later, Lehn pioneered supramolecular chemistry [24]. As such, research regarding artificial LHSs and efficient excited energy transfer system was initiated using covalently bonded molecular assemblies [method (a)]. Numerous studies have been conducted on method (a) using covalently linked porphyrin dimers [10, 25], trimers, tetramers, and dendrimers [7] with or without the combination of other kinds of dyes.

Following the establishment of supramolecular chemistry, method (b) was investigated intensively. Supramolecular architectures primarily depend on the interactions between molecules, such as coordination bonds, hydrogen bonds, van der Waals interactions, and electrostatic interactions. Since porphyrin derivatives have a center metal and/or hydroxyl group in their meso-positions, supramolecular porphryin networks have been studied $[8,17,26]$. The advantage of method (b) is the flexible structure owing to the supramolecular interactions. However, one disadvantage is the instability of the assembled structure. In natural photosynthesis, proteins play an important role in manipulating the supramolecular dye assembly and stabilizing the structure. Thus, the next step toward the realization of efficient artificial LHSs should be the induction of effective hosts that can manipulate the dye assembly and stabilize the assembled structures.

Accordingly, the use of inorganic host materials [method (c)] has been investigated. While efficient lightharvesting energy transfer is difficult in method (c), some works have reported excellent results with metalorganic frameworks [21] or periodic mesoporous organosilica [22]. My approach toward efficient artificial LHSs is based on method (c), in which clay nanosheets are used as the host material to build the 2D dye assembly using host (nanosheet)-guest (molecule) interactions. A clay nanosheet [27-31], saponite, is an attractive material characterized by (1) nanostructured flat sheets that can be formed by elements with large Clarke numbers (expressing the average content of the chemical elements in the earth), (2) negatively charged surfaces, (3) exfoliation or stacking ability of individual nanosheets in water, and (4) optical transparency in the visible light region in the exfoliated state when the particle size is small (ca. $<200 \mathrm{~nm}$ ). The unit structure of saponite is shown in Fig. 1. Saponite clay possesses layers consisting of 2:1 pairs of nanosheets with octahedral and tetrahedral microstructures. The typical chemical formula of saponite is $\left[\left(\mathrm{Si}_{7.20} \mathrm{Al}_{0.80}\right)-\left(\mathrm{Mg}_{5.97} \mathrm{Al}_{0.03}\right)\right.$ $\left.\mathrm{O}_{20}(\mathrm{OH})_{4}\right]^{-0.77}\left(\mathrm{Na}_{0.77}\right)^{+0.77}$. The isomorphous substitution of $\mathrm{Si}^{4+}$ by $\mathrm{Al}^{3+}$ in the tetrahedral layer produces anionic charges within the structure. The cation exchange capacity (CEC) is $0.997 \mathrm{meq} \mathrm{g}^{-1}$ and the theoretical surface area is $7.5 \times 10^{2} \mathrm{~m}^{2} \mathrm{~g}^{-1}$; thus, the average intercharge distance on the clay surface is calculated to be $1.2 \mathrm{~nm}$ on 


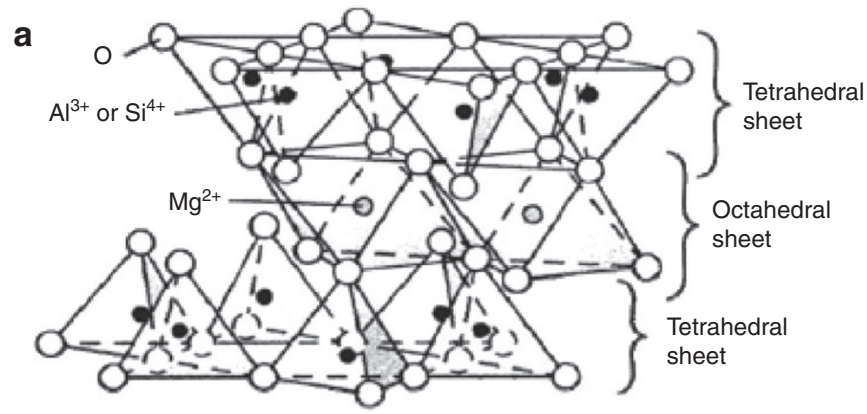

b

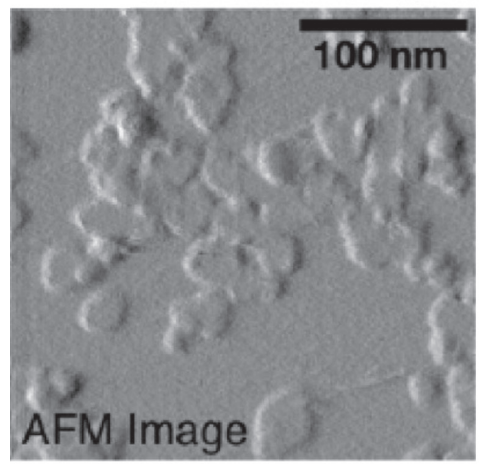

C

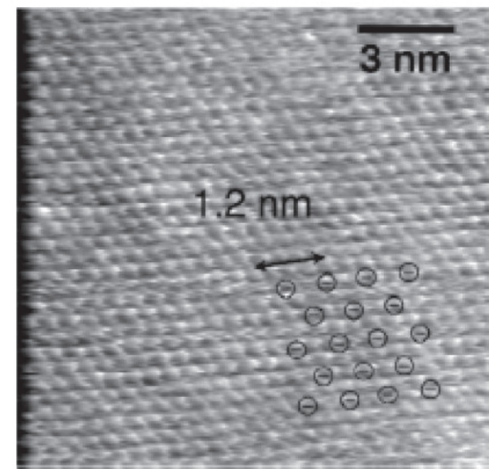

Fig. 1 (a) Structure of saponite having one octahedral layer sandwiched by two tetrahedral layers, AFM images of (b) clay particles and (c) the surface of a clay particle and the ideal distribution of anionic charge.

the basis of a hexagonal array. As seen in the AFM images in Fig. 1c, the clay particles have very flat surfaces over a wide area. Clay nanosheets are usually obtained as powders composed of large secondary particles. However, the stacked clay sheets can exfoliate to individual mononanosheets in water at low concentrations. The experiments and results presented in this short review have been carried out using exfoliated single nanosheets.

On the negatively charged surfaces, cationic dyes can be absorbed via electrostatic interactions. However, cationic organic molecules easily form aggregates mainly owing to hydrophobic interactions and van der Waals interactions, and thus it is difficult to control their 2D-alignment on the clay surface [28, 32, 33]. Since $\mathrm{H}$-aggregation and irregular aggregation drastically decreases the excited state lifetime, efficient photochemical reactions such as light-harvesting energy transfers have been regarded as impossible in clay/ dye complexes.

My group reported a novel mechanism to completely suppress dye aggregations on clay surfaces and to construct uniform 2D-alignments. Through systematic experiments using numerous cationic porphyrin derivatives, it has been found that a porphyrin molecule, which has a specific molecular structure, can be adsorbed on clay surfaces even under high-density conditions without concomitant aggregation (Fig. 2). Such nonaggregated alignment results from distance matching due to guest-host Coulombic interactions between the positively charged dye molecules and the negatively charged clay surface. This effect is termed the "sizematching effect [29]." In the case of tetra-cationic porphyrins, the average center-to-center intermolecular

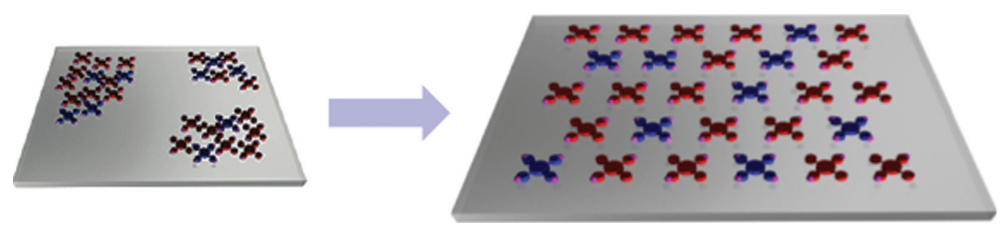

Fig. 2 Schematic representation of clay/dye supramolecular complex. 
distance was calculated to be $2.4 \mathrm{~nm}$ under saturated adsorption conditions on the basis of a hexagonal array [29]. This value is most interesting from the viewpoint of photochemistry, since the interaction between the transition moments of adjacent porphyrin molecules (aggregation) is negligible. However, interactions in the excited state are possible, and thus efficient photochemical reactions are expected. These findings persuaded us to examine photochemical reactions and light-harvesting energy transfers using this unique clay/ dye supramolecular system.

\section{Efficient energy transfer reaction between two porphyrin molecules on clay surfaces}

Excited-state energy-transfer reactions among the adsorbed porphyrins on the clay surface were examined [20]. In the first experiment, the maximum quantum yield of the energy transfer was $c a .35 \%$ [34] between the Zn porphyrin [zinc tetrakis( $N, N, N$-trimethylanilinium-4-yl) porphyrin] and the free base porphyrin [tetrakis(1methylpyridinium-4-yl) porphyrin] on the clay surface. In this clay/dye system, the quenching of the aggregated form was completely suppressed by the size-matching effect. Thus, the major factors that decreased the excited singlet energy-transfer efficiency were assumed to be self-fluorescence quenching processes [35] between porphyrins on the clay surface in the nonaggregated form and/or the segregation of the two dyes. The reaction conditions should be optimized in terms of the following aspects: (i) choosing appropriate porphyrins in which the self-fluorescence quenching is negligible [35], (ii) choosing porphyrins that do not form segregated structures [36], and (iii) adjusting the reaction conditions so that the adsorption density and the donor-acceptor ratio regulate the intermolecular distances [20].

The energy-transfer reaction from the excited singlet state of $m$-TMPyP [donor, tetrakis(1-methylpyridinium-3-yl) porphyrin] to the ground state of $p$-TMPyP [acceptor, tetrakis(1-methylpyridinium-4-yl) porphyrin] on the clay surface was investigated by steady-state and time-resolved fluorescence measurements. The porphyrin loading level vs. the cation exchange capacity (CEC) of the clay was adjusted from $0.05 \%$ to $90 \%$ by changing the concentration of the clay under a constant dye concentration $\left(1.0 \times 10^{-7} \mathrm{M}\right)$. The fluorescence spectra changed depending on the loading level of the porphyrin (Fig. 3). Clear isoemissive points were observed as indicated by the arrows in the figure. A decrease in the donor fluorescence and an increase in the acceptor fluorescence were observed as the dye loading increased.

In Fig. 4, the energy-transfer efficiencies $\left(\eta_{\mathrm{ET}}\right)$ are plotted vs. the porphyrin loading levels. The energytransfer efficiency increased as the porphyrin loading level increased, because the average intermolecular distance between the porphyrins decreased. Notably, the maximum energy-transfer efficiency reached $c a$.

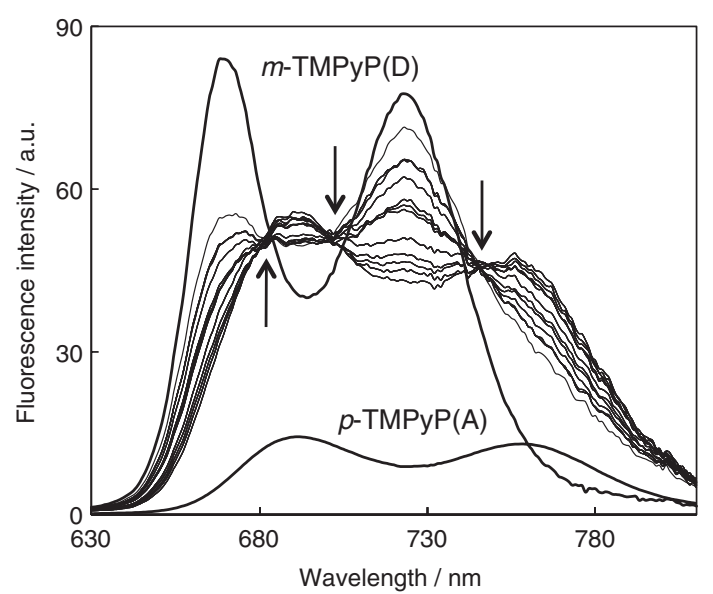

Fig. 3 The fluorescence spectra for $m$-TMPyP(D)/p-TMPyP(A)/clay complexes excited at $430 \mathrm{~nm}$ in aqueous solution. The porphyrin loading levels were set at $0.05-90 \%$ vs. CEC of the clay. The thick lines are individual fluorescence spectra of $m$-TMPyP(D)/clay and $p$-TMPyP(A)/clay complexes. The arrows indicate isoemissive points. Reprinted with permission from ref [20] (copyright 2011, American Chemical Society). 
$100 \%$ with $m$-TMPyP(D)/p-TMPyP(A) $=1 / 3,1 / 8$, and $1 / 15$ and the loading levels of porphyrin were $80 \%$ and $90 \%$ vs. CEC. The Förster-type energy transfer rate constant is inversely proportional to the sixth power of the intermolecular distance [23, 37]. The energy transfer efficiency increased on increasing the ratios of $p$ TMPyP on the clay surface, because the number of energy acceptors ( $p$-TMPyP) around the donor porphyrin molecules ( $m$-TMPyP) contributed to the frequency factor of the energy transfer rate. Accordingly, the adsorption of the porphyrins on the clay surface could be discussed. Generally, two kinds of dyes segregate from each other when the dyes coadsorb on the clay surface [38]. If the two kinds of porphyrins were coadsorbed on the clay surface with a segregated structure (Fig. 5a), then the energy-transfer efficiencies would not have reached $100 \%$ because the donor porphyrins surrounded by other donor porphyrins could not efficiently transfer the excited energy to the acceptor porphyrin. Therefore, as an example of the possible arrangements, the ideal arrangement of the coadsorbed porphyrins on the clay surface can be depicted as shown in Fig. 5b, where each donor molecule is surrounded by acceptor molecules. In the clay-porphyrin complexes, each dye has a monomeric adsorption on the clay surface with little interactions between the adsorbed porphyrins due to the size-matching effect. Thus, the integrated structure (Fig. 5b: a structure with a randomly adsorbed state) was realized in the present complexes.

The time-resolved fluorescence spectra of the $m$-TMPyP(D)/p-TMPyP(A)/clay complexes were examined in order to elucidate the details of the energy-transfer reaction. The ratio of donor/acceptor porphyrin [m-TMPyP(D)/p-TMPyP(A)] was $1 / 3$, and the total concentration of porphyrin was set at $90 \%$ loading vs. the CEC of the clay. Under such conditions, the $\eta_{\mathrm{ET}}$ value determined via steady-state fluorescence experiments was $c a .100 \%$, as shown in Fig. 4. The observed time-resolved fluorescence spectra for the $m$-TMPyP(D)/pTMPyP(A)/clay complex excited at $430 \mathrm{~nm}$ are shown in Fig. 6. The change in the spectral shape indicated the energy transfer from the excited donor porphyrin to acceptor porphyrin. At 4.0-4.2 ns, the obtained spectrum agreed well with that of $p$-TMPyP(A)/clay.

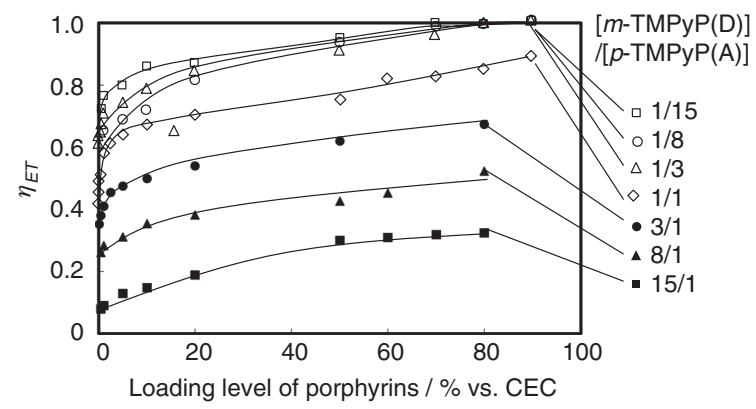

Fig. 4 The energy transfer efficiencies at various loading levels and ratios of porphyrins. $[m-\mathrm{TMPyP}(\mathrm{D})]+[p-\mathrm{TMPyP}(\mathrm{A})]=1.0 \times$

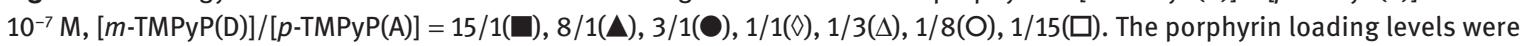
set at $0.05-90 \%$ vs. CEC of the clay in aqueous solution. Reprinted with permission from ref [20] (copyright 2011, American Chemical Society).

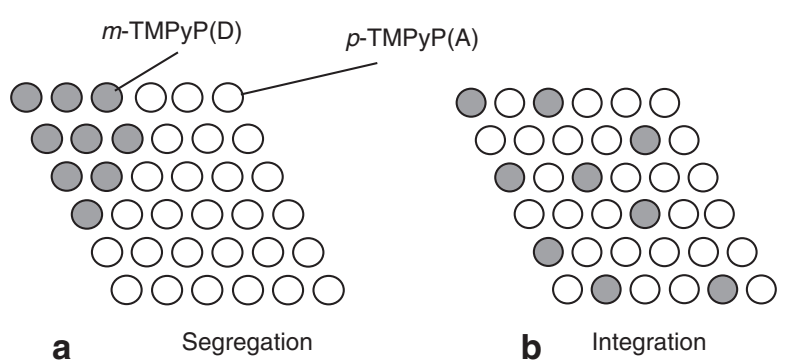

Fig. 5 Possible ideal images of segregation (a) and integration (b) adsorption structure of two kinds of porphyrins on the clay surface underm-TMPyP(D) / $p$-TMPyP(A) = 1/3. Reprinted with permission from ref [20] (copyright 2011, American Chemical Society). 
Fluorescence decay analysis for the $m$-TMPyP(D)/p-TMPyP(A)/clay complex was conducted in order to estimate the energy transfer rate constant $\left(k_{\mathrm{ET}}\right)$. In Fig. 7, the fluorescence decay curves for the $m$-TMPyP(D)/p-TMPyP(A)/clay complex between a) 635-655 $\mathrm{nm}$ and b) 770-790 $\mathrm{nm}$ are shown. The regions 635-655 and 770-790 nm mainly corresponds to the fluorescence of $m$-TMPyP(D) and $p$-TMPyP(A), respectively. As shown in Fig. 7a, the decay curve of $m$-TMPyP(D)/p-TMPyP(A)/clay at $635-655 \mathrm{~nm}$ was determined to be a double exponential decay. The lifetimes were calculated to be $0.4 \pm 0.1 \mathrm{~ns}\left(\tau_{1}\right)$ and $5.6 \pm 0.1$ ns $\left(\tau_{2}\right)$, respectively. The $\tau_{2}$ value agreed well with the $\tau_{\text {Acceptor }}$ value, and the decay curve did not contain any $\tau_{\text {Donor }}(7.4 \mathrm{~ns})$ component. These results suggested that $\tau_{1}$, the short lifetime component, was derived from the excited energy transfer from $m$-TMPyP(D) to the adjacent $p$-TMPyP(A) on the clay surface. In the case of the decay curve of $m$-TMPyP(D)/p-TMPyP(A)/clay complex at 770-790 nm (Fig. $7 \mathrm{~b}$ ), the rise and decay components, which were double exponentials, were observed. The lifetimes of the rise and decay components were calculated to be $0.4 \pm 0.1 \mathrm{~ns}\left(\tau_{3}\right)$ and $5.6 \pm 0.1 \mathrm{~ns}\left(\tau_{4}\right)$, respectively. The obtained lifetime value of rise component $\left(\tau_{3}\right)$ in the region $770-790 \mathrm{~nm}$ completely agreed with that of decay component $\left(\tau_{1}\right)$ in the region near $635-655 \mathrm{~nm}$. Thus, it can be concluded that $\tau_{1}$ and $\tau_{3}$ were the components derived from the energy transfer reaction.

From the short lifetime ( $\tau_{1}$ or $\tau_{3}=0.4 \pm 0.1 \mathrm{~ns}$ ) and lifetime of donor porphyrin $\left(\tau_{\text {Donor }}=7.4 \pm 0.1 \mathrm{~ns}\right)$, the energy transfer rate constant $k_{\mathrm{ET}}$ can be calculated to be $2.5 \pm 0.6 \times 10^{9} \mathrm{~s}^{-1}$ [23]. Notably, $k_{\mathrm{ET}}$ could be analyzed

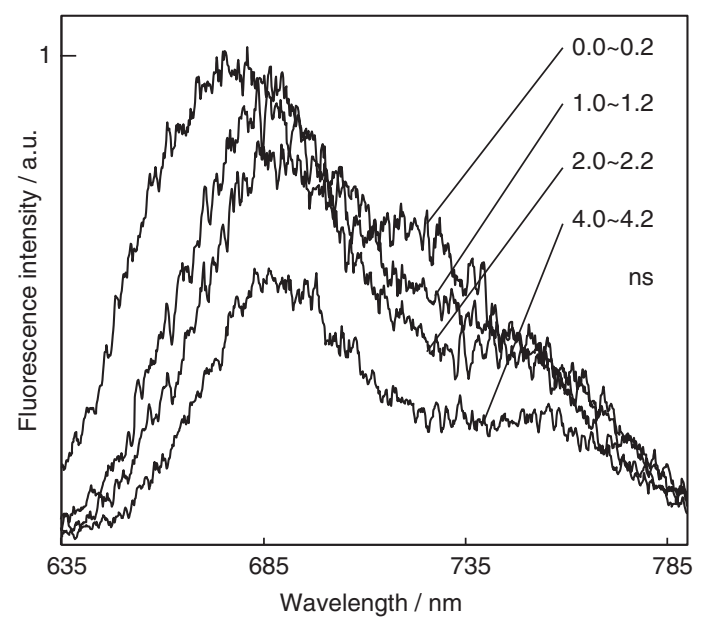

Fig. 6 (a) The time-resolved fluorescence spectra for $m$-TMPyP(D)/p-TMPyP(A)/clay complex at 0.0 0.2, 1.0 1.2, 2.0 2.2, 4.0 4.2 ns after excitation. The excitation wavelength was $430 \mathrm{~nm}$. The sum of dyes loadings was set at $90 \%$ vs. CEC of the clay, and $m$-TMPyP(D)/p-TMPyP(A) = 1/3. Reprinted with permission from ref [20] (copyright 2011, American Chemical Society).

a

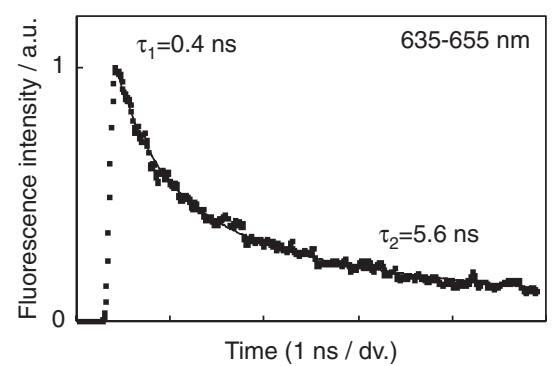

b

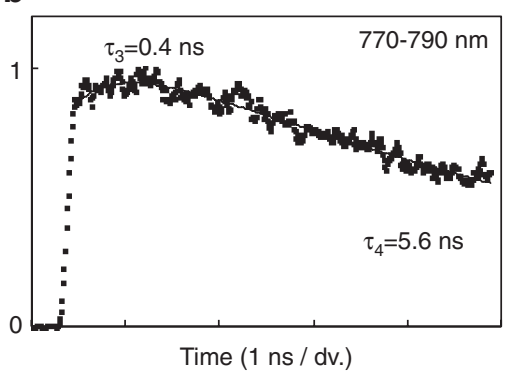

Fig. 7 The fluorescence decay profiles of $m$-TMPyP(D)/ p-TMPyP(A)/clay complex in the region of (a) 635-655 nm and (b) 770$790 \mathrm{~nm}$. The region of 635-655 nm mainly corresponds to the fluorescence of $m$-TMPyP(D)/clay, and the region of $770-790 \mathrm{~nm}$ almost corresponds to the fluorescence of $p$-TMPyP(A)/clay. The obtained values $(0)$ and the fitted curves (solid line) are shown. The conditions of samples were $90 \%$ dyes loadings vs. CEC of the clay, and $m$-TMPyP(D) / $p$-TMPyP(D) $=1 / 3$. Reprinted with permission from ref [20] (copyright 2011, American Chemical Society). 
only for one component. This strongly indicated that the porphyrin molecules did not aggregate and that the intermolecular distance was rather uniform on the clay surface under the saturated conditions. In usual photochemical reactions on solid surfaces [27,28], the photochemical behaviors of dyes are complicated due to the varying intermolecular distances including the formation of aggregates. In this system, the porphyrin molecules were strongly adsorbed on the clay surface owing to the size-matching effect, leading to uniform intermolecular distances.

The major factors leading to the realization of the $100 \%$ energy-transfer reaction were (i) suppression of aggregation that shortened the excited lifetime [29], (ii) suppression of self-fluorescence quenching due to excited electron transfer [35], and (iii) establishing the integrated adsorption structure of the two dyes on the clay surface [36]. These factors were controlled by the size-matching effect. Accordingly, it was presumed that this efficient energy-transfer system could be extended to a wide range of dyes that fulfill the size-matching effect. Generally, $100 \%$ energy-transfer efficiency is required for the construction of artificial LHSs involving multistep energy-transfer processes. Judging from the aforementioned results, clay/porphyrin complexes constitute very promising candidates for photochemical reactions such as in artificial LHSs.

\section{Artificial light-harvesting model utilizing wide range of visible light}

Nearly $100 \%$ energy transfer was successfully achieved in the previous chapter on porphyrin-porphyrin system [20]. The next step involved improving the light-harvesting ability of the clay/dye system [39]. We proposed that the performance of an artificial LHS should be evaluated in terms of the photon-harvesting efficiency $\left(\eta_{\mathrm{PH}}\right)$ and visible-light-harvesting efficiency $\left(\eta_{\mathrm{VLH}}\right)$. The former $\left(\eta_{\mathrm{PH}}\right)$ expresses the number of photons captured by one acceptor molecule and is defined experimentally as the donor/acceptor ratio when the total energy transfer efficiency is $50 \%$. The latter $\left(\eta_{\mathrm{VLH}}\right)$ expresses the availability of the visible region of sunlight and is defined as the percentage of this visible region $(380-780 \mathrm{~nm})$, in which the extinction coefficient of the light-harvesting molecules exceeds $10^{4} \mathrm{M}^{-1} \mathrm{~cm}^{-1}$. This extinction coefficient indicates that $90 \%$ of sunlight can be absorbed by the LHS with $1 \mu \mathrm{m}$ thickness and $1 \mathrm{M}$ concentration. High $\eta_{\mathrm{PH}}$ and $\eta_{\mathrm{VLH}}$ are required for constructing efficient artificial LHSs. Although we reported an almost $100 \%$ energy transfer reaction between two types of porphyrin dyes on the clay surface in the previous chapter [20], $\eta_{\mathrm{PH}}$ and $\eta_{\mathrm{VLH}}$ were small at 3 and $47.5 \%$, respectively. Thus, the next step toward the realization of clay-based artificial LHSs was to improve both $\eta_{\mathrm{PH}}$ and $\eta_{\mathrm{VLH}}$ by using appropriate dye combinations.

A new system was investigated using tetrakis(1-methylpyridinium-4-yl) porphyrin (Por) as the energy donor and tetramethyltetrapyridino [3,4-b: 3',4'-g: 3",4"-1: 3'", 4'"--q]porphyrazin (Pc) as the acceptor [39]. Due to the strong and wide absorption of Pc across the near IR region, the $\eta_{\mathrm{VLH}}$ of this Por(D)-Pc(A) system improved to $86 \%$, which was almost two times greater than that of the previous porphyrin-porphyrin system (47.5\%). Individual fluorescence spectra of the Por(D)/clay and $\mathrm{Pc}(\mathrm{A}) /$ clay complexes and the energy transfer sample of the $\operatorname{Por}(\mathrm{D}) / \mathrm{Pc}(\mathrm{A}) /$ clay complex are shown in Fig. 8. The shape of the fluorescence spectra of the energy transfer sample was the same as that of the acceptor fluorescence and the intensity increased. The fluorescence of the energy transfer sample did not include the Por(D) component. This indicated that the excited Por(D) molecules were completely deactivated by the energy transfer and/or other quenching processes such as self-quenching.

Energy transfer experiments were examined under $\operatorname{Por}(\mathrm{D}) / \mathrm{Pc}(\mathrm{A})=1 / 1-31 / 1$. The mol ratio of $\operatorname{Por}(\mathrm{D}) / \mathrm{Pc}(\mathrm{A})$ is expressed as $a / 1$ in Fig. 9. A large $a$ value indicates an efficient light-harvesting-type energy transfer in which only a few acceptors harvest the excitation energies from many donors. That is, the photon-harvesting efficiency $\left(\eta_{\mathrm{PH}}\right)$ tends to be high under high $a$ conditions. The energy transfer efficiency $\left(\eta_{\mathrm{ET}}\right)$ and self-quenching efficiency $\left(\phi_{\mathrm{AA}}\right)$ are plotted vs. $a$ in Fig. 9. When the ratio of Por(D) increased, $\phi_{A A}$ decreased, indicating that the self-quenching of $\mathrm{Pc}(\mathrm{A})$ was suppressed due to the decreased probability of adjacent $\mathrm{Pc}(\mathrm{A})$ molecules on the clay surface. The value of $\phi_{A A}$ became almost 0 at $a=15-31$, indicating that only the energy transfer proceeded and all other quenching processes were negligible at $a=15-31$. At $a=1-5, \eta_{\mathrm{ET}}$ was maintained at $100 \%$. The decrease of $\eta_{\mathrm{ET}}$ at $a=6-31$ would be due to the decreased probability of adjacent Por(D) and Pc(A) 


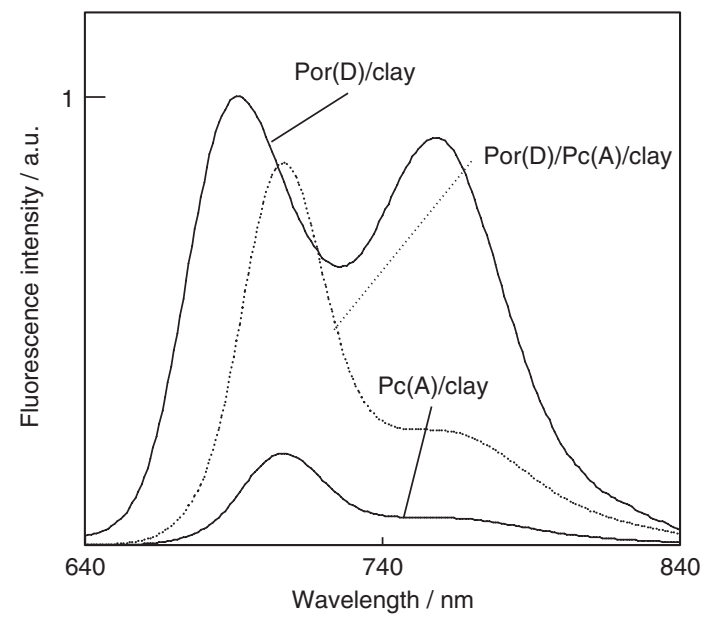

Fig. 8 Fluorescence spectra of $\operatorname{Por}(\mathrm{D}) /$ clay, $\mathrm{Pc}(\mathrm{A}) / \mathrm{clay}$ and $\mathrm{Por}(\mathrm{D}) / \mathrm{Pc}(\mathrm{A}) / \mathrm{clay}$ (dashed line) complexes excited at $450 \mathrm{~nm}$. The total dye loadings were set at $0.05 \%$ for $\operatorname{Por}(D) /$ clay and $\operatorname{Pc}(A) / c l a y$, and $90 \%$ for $\operatorname{Por}(D) / P c(A) / c l a y$ complex, respectively. The mol ratio of $\operatorname{Por}(D)$ to $\operatorname{Pc}(A)$ was 1 to 1 for $\operatorname{Por}(D) / \operatorname{Pc}(A) /$ clay complex. Reprinted with permission from ref [39] (copyright 2013, American Chemical Society).

molecules. Since Förster-type energy transfer rate constantsare inversely proportional to the sixth power of the intermolecular distance, an energy transfer from Por(D) to nonadjacent Pc(A) should be inefficient compared to that of an adjacent $\operatorname{Por}(\mathrm{D})-\mathrm{Pc}(\mathrm{A})$ pair. Judging from the hexagonal adsorption of the two types of dyes on the clay surface, the decreased $\eta_{\mathrm{ET}}$ was reasonable.

The photon-harvesting efficiency $\left(\eta_{\mathrm{PH}}\right)$ of the previous porphyrin-porphyrin system was small $(\sim 3)$ because of the small energy transfer rate constant $\left(k_{\mathrm{ET}}, 2.4 \times 10^{9} \mathrm{~s}^{-1}\right)$ owing to the small spectral overlap integral $\left(J, 3.9 \times 10^{-14} \mathrm{M}^{-1} \mathrm{~cm}^{3}\right)$. Specifically, the energy transfer was not efficient with excess donor concentrations, because the energy transfer between nonadjacent donor-acceptor pairs was nearly impossible. In the present system, since the spectral overlap between the fluorescence of Por(D) and the absorption of $\operatorname{Pc}(\mathrm{A})$ was rather large $\left(J, 1.1 \times 10^{-12} \mathrm{M}^{-1} \mathrm{~cm}^{3}\right), k_{\mathrm{ET}}$ was expected to be large. With the help of detailed time-resolved measurements and theoretical discussions, it was determined that there was a certain contribution of the energy

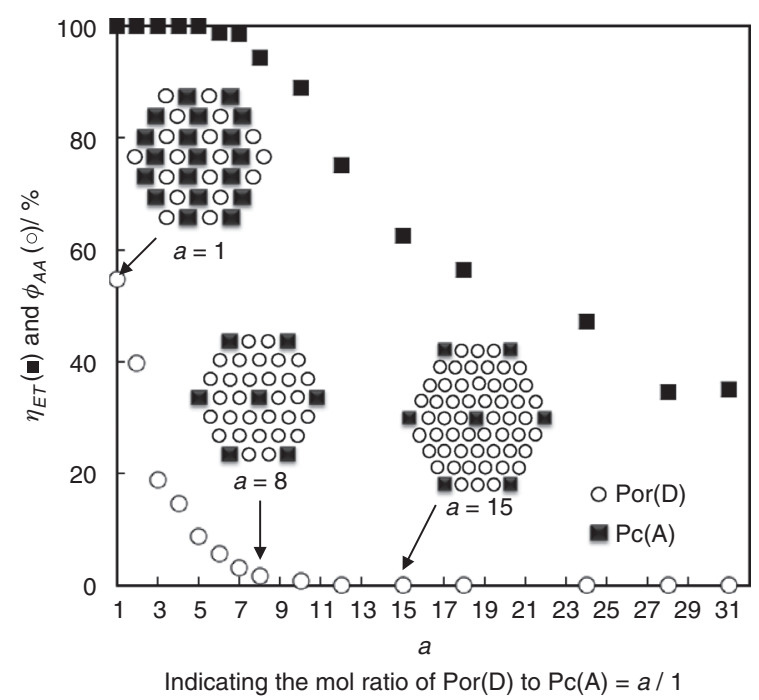

Fig. 9 Energy transfer efficiencies $\left(\eta_{\mathrm{ET}}, \square\right)$ and self-quenching efficiencies $\left(\phi_{\mathrm{AA}}, \mathrm{O}\right)$ in $\operatorname{Por}(\mathrm{D}) / \operatorname{Pc}(\mathrm{A}) / \mathrm{clay}$ complexes under various mol ratio conditions of $\operatorname{Por}(D)$ to $\operatorname{Pc}(A)$. The mol ratio of $\operatorname{Por}(D)$ to $\operatorname{Pc}(A)$ was modulated as $\operatorname{Por}(D) / \operatorname{Pc}(A)=1 / 1-31 / 1$. The total dye loading was set at $90 \%$ for $\operatorname{Por}(\mathrm{D}) / \mathrm{Pc}(\mathrm{A}) / \mathrm{clay}$ complex. The mol ratio of $\operatorname{Por}(\mathrm{D}) / \mathrm{Pc}(\mathrm{A})$ is expressed to $a / 1$. The schematic representations for possible idealized adsorption structures of $\operatorname{Por}(\mathrm{D}, \mathrm{O})$ and $\operatorname{Pc}(\mathrm{A}, \boldsymbol{\square})$ under $a=1,8,15$ conditions are depicted. Reprinted with permission from ref [39] (copyright 2013, American Chemical Society). 
transfer from excited Por(D) to nonadjacent Pc(A) (see the previous paper [39]). The value of $\eta_{\mathrm{PH}}$ of the present Por(D)-Pc(A) system was 21, as determined by Fig. 9, while that of our previous porphyrin-porphyrin system was about 3. Thus, this $\operatorname{Por}(\mathrm{D}) / \mathrm{Pc}(\mathrm{A}) /$ clay was much improved in terms of $\eta_{\mathrm{VLH}}$ and $\eta_{\mathrm{PH}}$.

\section{Artificial light-harvesting model utilizing wide variety of dyes}

As described in previous chapters, I demonstrated an efficient energy transfer reaction and the construction of an artificial light-harvesting model by employing the size-matching effect. In spite of their advantages, anionic clay nanosheets could only be used as hosts for cationic adsorbent molecules. Neutral molecules such as aromatics do not adsorb on these surfaces. I also reported a strategy to overcome this problem. The strategy involved a supramolecular approach in which a cationic organic host system that included a neutral guest molecule was adsorbed on anionic clay sheets [40, 41]. Such an approach would expand the utility of the size-matching effect to neutral donor-acceptor systems.

A cavitand with eight ammonium groups (octa amine, OAm [42]) was used as the host for neutral organic molecules (Fig. 10). Strong Coulombic attractions between the ammonium groups and the anionic surface of the clay anchored OAm to the clay nanosheets. Since OAm is known to form capsular assemblies with organic neutral guest molecules, we explored the possibility of energy transfer between two OAm capsules, one containing a donor molecule (pyrene, 2) and another encapsulating an acceptor molecule (2-acetylanthracene, 3) adsorbed on a clay nanosheet. The efficient energy transfer between 2@OAm ${ }_{2}^{16+}$ (pyrene encapsulated within two molecules of $\left.\mathrm{OAm}^{8+}\right)$ and 3@OAm ${ }_{2}^{16+}$ (2-acetylanthracene encapsulated within two molecules of $\mathrm{OAm}^{8+}$ ) in water adsorbed on saponite nanosheets is demonstrated in this chapter. Notably, the donor and acceptor aromatic molecules are insoluble in water and they could be solubilized only in the presence of OAm; neither molecule was adsorbed on the saponite surface in the absence of OAm. Thus, the use of a combination of clay and OAm allowed us to overcome these problems and provided new opportunities to explore supramolecular assemblies in light-harvesting applications.

Guests 2 and 3 were encapsulated within $\mathrm{OAm}^{8+}$ in an aqueous solution under acidic conditions ( $\mathrm{pH}$ 2) according to the previously reported procedure [40]. Inclusion and the ratio of the host to guest complex in each case were inferred by ${ }^{1} \mathrm{H}-\mathrm{NMR}$ titration experiments. Under the experimental conditions $(\mathrm{pH} \sim 2)$ used in this study, I believe that all eight triethylamine groups of OAm remained protonated. $\mathrm{OAm}^{8+}$ and $\mathrm{OAm}_{2}{ }^{16+}$ indicate the protonated OAm (1) with 8 and 16 cationic charges under acidic conditions, respectively. The 1:2 guest-host complexes of $\mathbf{2}$ and $\mathbf{3}$ with $\mathrm{OAm}^{8+}$ were adsorbed on saponite clay nanosheets by stirring the aqueous clay suspension with guest@OAm ${ }_{2}^{16+}$ at $\mathrm{pH} \sim 2$. By altering the concentration of clay and using the hexagonal array under the maximum adsorption conditions, it was found that both $2 @ 0 \mathrm{Am}_{2}{ }^{16+}$ and 3@OAm ${ }_{2}^{16+}$ adsorbed onto the clay surface and the center-to-center distance between cavitands were $2.4 \mathrm{~nm}$ (Fig. 11). Since the stabilities of these cavitand were investigated by absorption and fluorescence measurements, the singlet-singlet energy transfer between 2@OAm ${ }_{2}^{16+}$ and $3 @ 0 A m_{2}{ }^{16+}$ on the clay surface was examined.

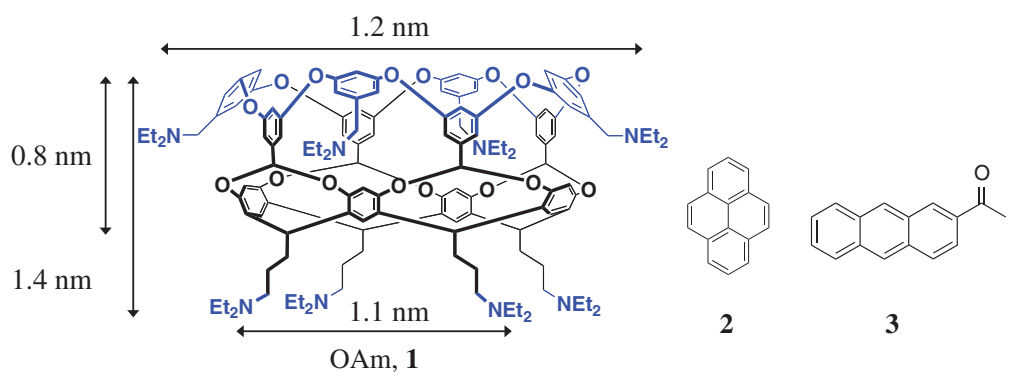

Fig. 10 Structures of octa amine cavitand (OAm, 1), pyrene (2) and 2-acetylanthracene (3). Reprinted with permission from ref [40] (copyright 2013, American Chemical Society). 


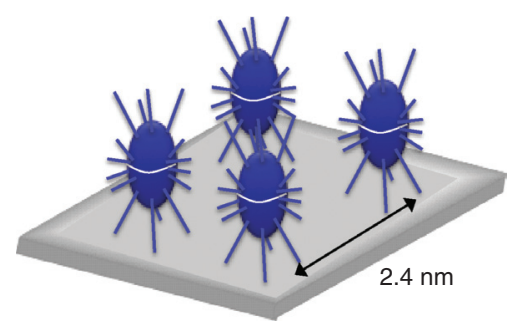

Fig. 11 Plausible adsorption structure model of guest@OAm ${ }_{2}^{16+}$ on the clay surface under the maximum adsorption condition. Reprinted with permission from ref [40] (copyright 2013, American Chemical Society).

The singlet-singlet energy transfer from the excited singlet state of $\mathbf{2} @ 0 \mathrm{Am}_{2}{ }^{16+}$ to the ground state of $\mathbf{3} @$ $\mathrm{OAm}_{2}{ }^{16+}$ on the clay surface was examined by monitoring the fluorescence intensity and lifetime of the donor and the fluorescence intensity of the acceptor. The excitation wavelength was set at $335 \mathrm{~nm}$ for all experiments. The fluorescence spectra of $\mathbf{2} @ O \mathrm{Am}_{2}{ }^{16+} \cap$ clay and $\mathbf{3} @ \mathrm{OAm}{ }_{2}{ }^{16+} \cap$ clay and $\left(\left\{\mathbf{2} @ \mathrm{OAm}{ }_{2}{ }^{16+}+\mathbf{3} @ \mathrm{OAm}{ }_{2}{ }^{16+}\right\} \cap\right.$ clay) are shown in Fig. 12(a). With increased loading levels, the fluorescence intensity of donor $\mathbf{2}$ decreased while that of acceptor $\mathbf{3}$ increased. This suggested the possibility of a singlet-singlet energy transfer from excited $\mathbf{2}$ to the ground state of $\mathbf{3}$. Because the fluorescence spectra were relatively well differentiated, the energy transfer efficiency $\left(\eta_{\mathrm{ET}}\right)$ could be estimated by analyzing the fluorescence spectra according a previously reported method [20]. The energy transfer and the quenching efficiencies were determined to be $85 \%$ and $0 \%$, respectively. The quenching process is typically caused by an electron transfer and/or enhanced thermal deactivation due to the collision between neighboring guests. The estimated $0 \%$ quenching efficiency was consistent with the fluorescence measurements of the individual systems. A plot of $\eta_{\mathrm{ET}} \mathrm{vs}$. the loading levels of the guests is provided in Fig. 12(b). As expected owing to the decreased intermolecular distance, $\eta_{\mathrm{ET}}$ increased with the loading level.

To probe whether the observed energy transfer was static or dynamic, time-resolved fluorescence measurements were carried out. The excitation and detection wavelengths were set at 335 and $360 \mathrm{~nm}$, which corresponded to the $\lambda_{\max }$ of the absorption and fluorescence of 2@OAm ${ }_{2}^{16+}$, respectively. The fluorescence of 2@ $\mathrm{OAm}_{2}{ }^{16+} \cap$ clay in the presence and absence of $3 @ 0 \mathrm{Am}_{2}{ }^{16+}$ showed a single exponential decay [Fig. 12 (c)]. Most importantly, the lifetime decreased from $350 \mathrm{~ns}$ (2@OAm ${ }_{2}^{16+} \cap$ clay) to $60 \mathrm{~ns}$ for $\left\{\mathbf{2} @ \mathrm{OAm}_{2}{ }^{16+}+\mathbf{3} @ \mathrm{OAm}_{2}{ }^{16+}\right\} \cap$ clay. From these values, the energy transfer rate constant $k_{\mathrm{ET}}$ was calculated to be $1.4 \times 10^{7} \mathrm{~s}^{-1}$. The monoexponential decay of the donor excited state suggested that the adsorption distribution of $\left\{\mathbf{2} @ 0 \mathrm{Om}_{2}{ }^{16+}+\mathbf{3} @\right.$ $\left.\mathrm{OAm}_{2}{ }^{16+}\right\}$ on the clay surface was uniform and they were not segregated (Fig. 5). In this study, since the guest
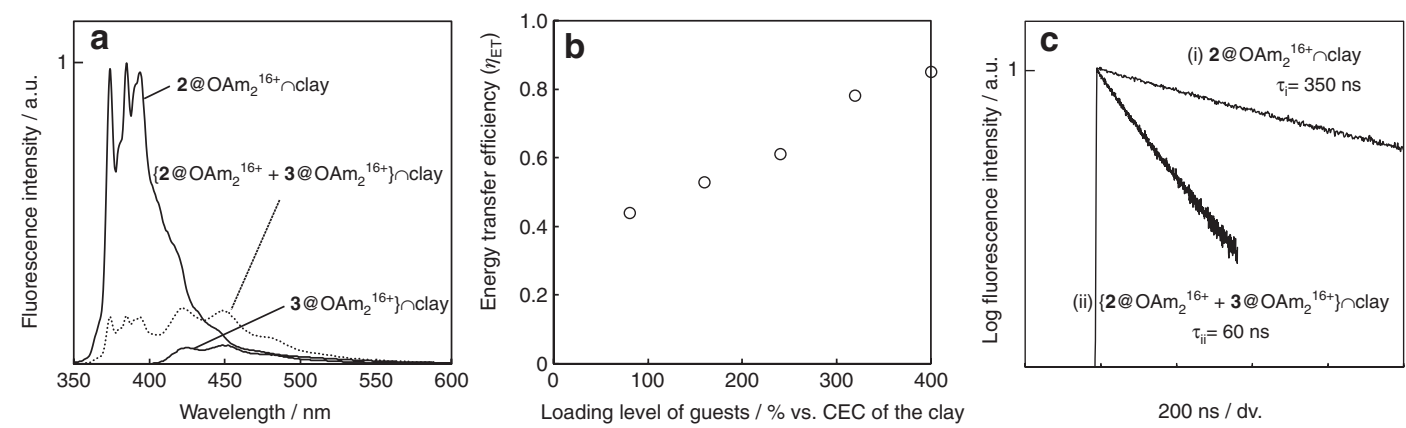

Fig. 12 (a) Fluorescence spectra for $\left\{2 @ 0 \mathrm{Am}_{2}{ }^{16+}+3 @ 0 \mathrm{Am}_{2}{ }^{16+}\right\} \cap$ clay complex and respective $2 @ 0 \mathrm{Am}_{2}{ }^{16+} \cap$ clay and $3 @ 0 \mathrm{Am}{ }_{2}{ }^{16+} \cap c l a y$ complexes. The excitation wavelength was $335 \mathrm{~nm}$. The loading levels were set at $400 \% \mathrm{vs}$. CEC of the clay for all samples. (b) The obtained values of energy transfer efficiencies $\left(\eta_{\mathrm{ET}}\right)$ at various loading levels of guests. The dye loadings were set at 80-400\% vs. CEC of the clay. (c) Nano-second time-resolved fluorescence decays for (i) 2@OAm ${ }^{16+} \cap$ clay and (ii) $\left\{2 @ 0 A m_{2}{ }^{16+}+\right.$ $\left.3 @ 0 m_{2}{ }^{16+}\right\} \cap$ clay (energy transfer sample). The excitation and detected wavelengths were set at $335 \mathrm{~nm}$ and $360 \mathrm{~nm}$, which are the $\lambda_{\max }$ of the absorption and fluorescence of $2 @ 0 A m_{2}{ }^{16+}$, respectively. The loading levels were set at $400 \%$ vs. CEC of the clay for all samples. Reprinted with permission from ref [40] (copyright 2013, American Chemical Society). 
molecules are encapsulated within an organic capsule, no direct interactions between the guest molecules were expected. Externally, all guest-included capsules had the same characteristics (positively charged) and their distribution on the clay surface would not be controlled by what was inside the capsule. This was the most likely reason for the rather uniform distribution of $2 @ \mathrm{OAm}_{2}{ }^{16+}$ and $3 @ \mathrm{OAm}{ }_{2}{ }^{16+\}}$ on the clay surface.

\section{Summary and perspective}

Herein, I presented my work regarding a novel approach toward the realization of artificial LHSs using hostguest supramolecular assemblies. The realization of applicable and efficient artificial LHSs requires further improvements such as an effective connection between the LHS and reaction center complexes, the utilization of a wide range of sunlight, and the high stability of the assembled structure. The connection to the reaction center complex is the most pressing concern. The use of the clay/dye system can overcome certain limitations. Since the formation of the clay/dye complex depends on host-guest interactions, it is easy to combine and exchange numerous dyes on one nanosheet. Thus, the efficient utilization of the entire visible light is possible when four or five types of dyes are used. Moreover, a highly stable dye organization is expected due to the high durability of the clay nanosheet, and thus enables the co-arrangement of both light-harvesting molecules and reaction center complex on the same surfaces.

While the limitations described above may be improvable by the today's knowledge, there are still large limitations for artificial light-harvesting system. One largest limitation for bio-mimicking light-harvesting system is to reproduce the amazing abilities of natural system, for example, the dissipation of excess excitation energy under much strong sunlight condition to avoid the decomposition of proteins by reactive oxygen spices. There is still no report to realize this system artificially, and I am always fascinated by the beautifulness and excellence of natural photosynthetic systems.

Artificial light-harvesting system has been much improved in the last two decades, however we still have limitations that have to be overcome. I believe that the findings presented here will contribute to the construction of efficient photochemical reaction systems in supramolecular host-guest assemblies as a step toward achieving artificial photosynthesis.

Acknowledgments: Y.I. thanks for JSPS Research Fellowships DC1 and PD from the Japan Society for the Promotion of Science, and JSPS KAKENHI Grant Number 26790002.

\section{References}

[1] H. Inoue, T. Shimada, Y. Kou, Y. Nabetani, D. Masui, S. Takagi, H. Tachibana. ChemSusChem 4, 173 (2011).

[2] A. Kimoto, K. Yamauchi, M. Yoshida, S. Masaoka, K. Sakai. Chem. Commun. 48, 239 (2011).

[3] H. Takeda, O. Ishitani. Coord. Chem. Rev. 254, 346 (2010).

[4] Y. Umena, K. Kawakami, J.-R. Shen, N. Kamiya. Nature 473, 55 (2012).

[5] D. Voet, J. G. Voet. Biochemistry. New York, Wiley, 1995.

[6] G. McDermott, S. M. Prince, A. A. Freer, A. M. Hawthornthwaite-Lawless, M. Z. Papiz, R. J. Cogdell, N. W. Isaacs. Nature 374, 517 (1995).

[7] R. Ziessel, A. Harriman. Chem. Commun. 47, 611 (2011).

[8] M. K. Panda, K. Ladomenou, A. G. Coutsolelos. Coord. Chem. Rev. 256, 2601 (2012).

[9] M. R. Wasielewski. Acc. Chem. Res. 42, 1910 (2009).

[10] Y. Nakamura, N. Aratani, A. Osuka. Chem. Soc. Rev. 36, 831 (2007).

[11] P. D. Frischmann, K. Mahata, F. Würthner. Chem. Soc. Rev. 42, 1847 (2013).

[12] K. V. Rao, K. K. R. Datta, M. Eswaramoorthy, S. J. George. Chem. Eur. J. 18, 2184 (2012).

[13] H. S. Cho, D. H. Jeong, M.-C. Yoon, Y. H. Kim, Y.-R. Kim, D. Kim, S. C. Jeoung, S. K. Kim, N. Aratani, H. Shinmori, A. Osuka. J. Phys. Chem. A 105, 4200 (2001).

[14] A. Uetomo, M. Kozaki, S. Suzuki, K.-I. Yamanaka, O. Ito, K. Okada. J. Am. Chem. Soc. 133, 13276 (2011).

[15] J.-S. Hsiao, B. P. Krueger, R. W. Wagner, T. E. Johnson, J. K. Delaney, D. C. Mauzerall, G. R. Fleming, J. S. Lindsey, D. F. Bocian, R. J. Donohoe. J. Am. Chem. Soc. 118, 11181 (1996). 
[16] Y. Kobuke. Eur. J. Inorg. Chem. 2006, 2333 (2006).

[17] H. Tamiaki. Coordin. Chem. Rev. 148, 183 (1996).

[18] T. Miyatake, H. Tamiaki, A. R. Holzwarth, K. Schaffner. Photochem. Photobiol. 69, 448 (1999).

[19] S. Fukuzumi, K. Saito, K. Ohkubo, T. Khoury, Y. Kashiwagi, M. A. Absalom, S. Gadde, F. D’Souza, Y. Araki, O. Ito, M. J. Crossley. Chem. Commun. 47, 7980 (2011).

[20] Y. Ishida, T. Shimada, D. Masui, H. Tachibana, H. Inoue, S. Takagi. J. Am. Chem. Soc. 133, 14280 (2011).

[21] H.-J. Son, S. Jin, S. Patwardhan, S. J. Wezenberg, N. C. Jeong, M. So, C. E. Wilmer, A. A. Sarjeant, G. C. Schatz, R. Q. Snurr, O. K. Farha, G. P. Wiederrecht, J. T. Hupp. J. Am. Chem. Soc. 135, 862 (2013).

[22] S. Inagaki, O. Ohtani, Y. Goto, K. Okamoto, M. Ikai, K.-I. Yamanaka, T. Tani, T. Okada. Angew. Chem. Int. Ed. 48, 4042 (2009).

[23] T. Förster. Discuss. Faraday Soc. 27, 7 (1959).

[24] J. M. Lehn. Pure Appl. Chem. 50, 871 (1978).

[25] D. Holten, D. F. Bocian, J. S. Lindsey. Acc. Chem. Res. 35, 57 (2002).

[26] H. Tamiaki, M. Amakawa, Y. Shimono, R. Tanikaga, A. R. Holzwarth, K. Schaffner. Photochem. Photobiol. 63, 92 (1996).

[27] T. Shichi, K. Takagi. J. Photochem. Photobiol. C: Photochem. Rev. 1, 113 (2000).

[28] F. López Arbeloa, V. Martínez Martínez, T. Arbeloa, I. López Arbeloa. J. Photochem. Photobiol. C: Photochem. Rev. 8, 85 (2007).

[29] S. Takagi, T. Shimada, Y. Ishida, T. fujimura, D. Masui, H. Tachibana, M. Eguchi, H. Inoue. Langmuir 29, 2108 (2013).

[30] T. Okada, Y. Ide, M. Ogawa. Chem. Asian J. 7, 1980 (2012).

[31] J. K. Thomas. Chem. Rev. 93, 301 (1993).

[32] J. Bujdák. Appl. Clay Sci. 34, 58 (2006).

[33] J. W. Davis, M. S. Kahl, T. D. Golden. J. Appl. Polym. Sci. 40141 (2013).

[34] Y. Ishida, T. Fujimura, D. Masui, T. Shimada, H. Tachibana, H. Inoue, S. Takagi. Clay Sci. 15, 169 (2011).

[35] Y. Ishida, T. Shimada, H. Tachibana, H. Inoue, S. Takagi. J. Phys. Chem. A 116, 12065 (2012).

[36] Y. Ishida, D. Masui, H. Tachibana, H. Inoue, T. Shimada, S. Takagi. ACS Appl. Mater. Interfaces 4, 811 (2012).

[37] K. F. Wong, B. Bagchi, P. J. Rossky. J. Phys. Chem. A108, 5752 (2004).

[38] P. K. Ghosh, A. J. Bard. J. Phys. Chem. 88, 5519 (1984).

[39] Y. Ishida, T. Shimada, S. Takagi. J. Phys. Chem. C 117, 9154 (2013).

[40] Y. Ishida, R. Kulasekharan, T. Shimada, S. Takagi, V. Ramamurthy. Langmuir 29, 1748 (2013).

[41] Y. Ishida, R. Kulasekharan, T. Shimada, V. Ramamurthy, S. Takagi. J. Phys. Chem. C 118, 10198 (2014).

[42] R. Kulasekharan, V. Ramamurthy. Org. Lett. 13, 5092 (2011). 